\title{
Recomendaciones para la confección de un corpus oral válido para el análisis fonético
}

\author{
Suggestions for building an accurate oral corpus to phonetics \\ analysis
}

Nuria Polo Cano

Universidad Nacional de Educación a Distancia (UNED), España

\begin{abstract}
Resumen: A menudo se cometen errores en la recogida y tratamiento de datos para la confección de un corpus oral, que en ocasiones pueden llegar hasta imposibilitar un posible análisis fonético posterior. Para evitar esto se proponen en estas páginas una serie de consejos previos relativos a los participantes, a las grabaciones y a las herramientas de construcción del corpus; todo ello con la idea de aconsejar al investigador a la hora de construir un corpus oral. Estos consejos le ayudarán a construir un corpus que permita el análisis fonético adecuado con los estándares de calidad científica actuales.
\end{abstract}

Palabras clave: corpus orales, análisis fonético, habla real, grabaciones, explotación de los datos.

Abstract: Mistakes are often made when an oral corpus is collected and sometimes these mistakes could make impossible a future phonetic analysis of the data. To avoid this happen some advices are proposed in this paper regarded to participants, to recordings, and to available tools in order to build an oral corpus. The purpose of this paper is to advice future researchers in building this kind of corpus. These advices will help them to build an accurate corpus to phonetics analysis following current scientific quality standards.

Keywords: oral corpus, analysis of phonetics, natural speech, recordings, data-mining.

Sobre la creación de corpus se ha escrito mucho, tanto que incluso dentro de la lingüística se viene desarrollando desde hace varios años ya una nueva rama denominada lingüística de corpus (McEnery y Wilson, 1996; Kennedy, 1998; Kock, 2001; entre muchos otros). El objetivo de estas páginas no es resumir la historia de los corpus, ni explicar su desarrollo, ni recopilar lo distintos tipos existentes ${ }^{\prime}$; el objetivo de estas páginas es llamar la atención sobre ciertos aspectos de la confección de un corpus para que pueda ser apto para el análisis del habla oral y, por tanto, para el análisis fonético. Tanto es así que recientemente se ha publicado un manual específico sobre la fonología de corpus (Durand, Gut y Kristoffersen, 2014).

Los aspectos que se abordan en estas páginas se refieren tanto a las recomendaciones que atañen a los participantes como a las

\footnotetext{
' Torruella y Llisterri (1999) ofrecen un repaso de los distintos tipos de corpus.
} 
recomendaciones que conciernen al modo en el que se realiza la grabación, ya que ambas influyen directamente en la validez del corpus para realizar análisis fonéticos. Asimismo se repasan algunos de los sistemas habituales para construir las bases de datos.

\section{Recomendaciones sobre los participantes}

El primer paso para crear un corpus es la búsqueda de informantes. En este proceso puede ocurrir una posible desconfianza ante el investigador, así que este se presentará y dirá siempre quién es, a qué institución pertenece o quién financia la investigación (esta información además puede abrir muchas puertas), los objetivos generales del estudio y qué es lo que se va a realizar: grabaciones de vídeo, audio, una entrevista, etc.

En numerosas ocasiones, los investigadores acuden a amigos o conocidos para que participen en la confección del corpus, ya que el desconocimiento total del investigador en muchos casos dificulta la búsqueda de hablantes voluntarios para las entrevistas, por no decir que las imposibilita en una gran cantidad de las veces. Para evitar este primer rechazo López Morales (1994: 87) propone, por ejemplo, en zonas rurales resulta "útil acudir a autoridades civiles, religiosas o educativas en busca de cooperación para lograr una atmósfera positiva y una actitud propicia".

Una vez que se cuenta con los informantes, como en cualquier otro tipo de investigación se debe conseguir siempre un consentimiento informado al principio del estudio y sin este no se puede comenzar la recogida de datos. En él, se explica a los participantes los objetivos del trabajo y que sus datos personales serán anónimos y se tratarán con el máximo respeto. Además, se les tiene que advertir de que van a ser grabados y de que esa información será accesible en abierto a toda la comunidad científica ${ }^{2}$. Por ello, antes de comenzar cualquier estudio el investigador se asegurará de que los participantes han entendido tanto sus derechos como sus deberes y tratará de resolver las posibles dudas que les puedan surgir. De todas formas, no es necesario contar todo el objetivo de la investigación, si se intuye que ello puede influir negativamente en la muestra de habla que se pretende recoger.

En este sentido, para proteger su privacidad, aunque se recoja el nombre de la persona que se ha grabado, este no aparecerá ni en la grabación oral ni en la posterior transcripción escrita. Además, si en un momento dado cuenta algo que sea demasiado personal, alguna anécdota íntima o una opinión delicada, cuyo contenido, la mayoría de las veces es irrelevante en investigaciones lingüísticas, quizás el investigador podrá plantearse apagar la grabadora y continuar después o eliminar esa parte de la grabación en la confección de su corpus. Es fundamental, por tanto, que los informantes puedan expresarse libremente, sin sentimientos inhibidores. En definitiva, el investigador mostrará siempre una actitud respetuosa hacia ellos.

Sobra decir que en ningún momento se puede esconder la grabadora para realizar cualquier estudio. Algunos investigadores en épocas pasadas han

\footnotetext{
2 En el pasado los corpus no se publicaban, ahora la tendencia es, en general, que estén a disposición de la comunidad científica en abierto gracias al soporte que ofrece Internet.
} 
utilizado las denominadas grabaciones secretas, ocultando cualquier dispositivo de grabación para que el participante no se diera cuenta de que estaba siendo grabado y hablase libremente, sin distorsionar su manera idiosincrásica de hablar. Sin embargo, hoy en día no se utiliza esta práctica, ya que se ha demostrado que los informantes se olvidan de la grabadora a los pocos minutos de empezar a hablar $y$, de hecho, hacer uso de este tipo de prácticas se ha convertido en una acción ilegal y penada por la ley actualmente.

Una vez que se han seleccionado los participantes del corpus, lo más recomendable es disponer de una habitación sin ruidos para realizar la toma de datos. Se intentará crear un espacio que provoque un clima de confianza y procurar que no se produzcan interrupciones. Es importante que antes de que se comience a grabar se explique a la persona que va a ser grabada que no se puede comer durante la grabación, que no se puede levantar, por ejemplo, para ir a saludar a alguien que ha visto pasar, y que apague el teléfono móvil, pues creará interferencias en la señal en caso de que le llamen. Por ello, es aconsejable informar del tiempo que va a durar la grabación para que el informante disponga del tiempo suficiente para realizarlas, ya que pueden durar desde 10-15 minutos hasta una hora ${ }^{3}$. Es recomendable que se eviten las horas de las comidas o las horas de preparación de las mismas. En todo caso no está demás tener un vaso de agua cerca para que los participantes beban un poco si se sienten cansados.

No es conveniente que se realice la grabación en la calle o en un espacio abierto, porque puede haber mucho ruido, puede haber viento o puede suponer distracciones para la persona que está hablando. Para cualquier otro tipo de corpus, que haya cierto ruido o viento no influye en la transcripción si el habla es inteligible, como para el análisis de la sintaxis, por ejemplo, pero para un análisis fonético un ruido de fondo o el viento invalidarán ese trozo de la grabación.

Normalmente, y sobre todo en los corpus que serán utilizados para el análisis fonético, las grabaciones se realizan de forma individual, a una única persona. De la misma forma, para este tipo de estudios o análisis de voz, antes de comenzar la grabación se tienen que calibrar los micrófonos que se vayan a usar cada día, porque las condiciones de grabación pueden cambiar con el ambiente (Svec y Granqvist, 2010).

Cuando lo que interesa grabar son conversaciones -situaciones reales-, por razones sociolingüísticas o pragmáticas, donde un grupo de personas interactúan, a la hora de analizarlas habrá que tener en cuenta la superposición y el solapamiento de voces, hecho característico de las conversaciones espontáneas. En este tipo de situaciones, si se va a realizar un análisis fonético, también se prestará atención, cuando se transcriban los datos, al tono de voz, a las vacilaciones, a la intensidad, a los silencios, a las pausas, etc.

El investigador tratará de comunicarse con naturalidad para que el informante se olvide de que está siendo grabado. Uno de los errores comunes y que el

\footnotetext{
${ }^{3}$ No es recomendable que duren más de una hora para evitar agotar al informante.
} 
entrevistador o investigador evitará cometer a la hora de realizar entrevistas es participar demasiado y no permitir que el informante diga lo que quiere decir o interrumpirle y hablar por encima de él, ya que no se podrá realizar un análisis fonético si el habla está superpuesta.

No obstante, aunque demos libertad a los hablantes, ante la presencia de extraños como son los investigadores o la propia grabadora, estos pueden sentirse cohibidos y limitarse a responder con monosílabos a las preguntas. Para evitar esta situación y la consiguiente pérdida de tiempo por ambas partes, Silva-Corvalán (2001: 53) sugiere, por ejemplo, un contacto inicial previo con los hablantes, para que se familiaricen con el investigador antes de ser grabados; el problema de esta práctica es que requiere mucho más tiempo. Por su parte, López Morales (1994: 78-79) aconseja que el entrevistador sea un conocido o amigo, o al menos perteneciente a la misma comunidad de habla. Y propone, además, intentar involucrar emocionalmente al informante, de manera que la emotividad neutralice la participación de su conciencia lingüística. De esta forma se consigue desinhibir al entrevistado y que relate, por ejemplo, situaciones de su pasado. Sin embargo, si se va a realizar un análisis de la entonación es aconsejable que se evite el uso de las emociones, pues estas afectarán la curva melódica natural y un discurso cargado de emociones no servirá entonces a los propósitos de ese corpus.

En el caso de que investigador e informante pertenezcan a comunidades de habla diferentes, un habla diatópica o diastráticamente diferente por parte del entrevistador puede hacer que el informante se amolde o elimine sus rasgos idiosincrásicos y trate de copiar el habla del entrevistador, por ejemplo, tratando de utilizar unos rasgos fonéticos estándares. Asimismo a menudo las respuestas a tareas lingüísticas estimulan la conciencia lingüística de los que responden $y$, por tanto, pueden contestar con la forma que los informantes creen que deberían, la más culta, y la que el lingüista merecería por ser el estudioso de la lengua (Silva-Corvalán: 2001: 63). Para evitar que esto ocurra no es necesario contar a los informantes que el trabajo es lingüístico, por ejemplo.

En cualquier caso, las grabaciones se planifican previamente y el tipo de preguntas o entrevistas que se van a realizar se tienen que estructurar de antemano. Es aconsejable formular preguntas abiertas, dirigidas, a las que el informante va contestando y se pueden ir introduciendo temas generales, para que el hablante vaya hablando de forma semidirigida. Así, si se deja que el informante hable de forma libre, la conversación se tenderá a focalizar sobre un tema que el investigador establezca de antemano para que todos los participantes aporten respuestas similares $y$, por tanto, comparables. Por ello, hay que seleccionar previamente las preguntas que se van a realizar, las cuales se recomienda que sean claras, de fácil comprensión y concisas. Además, se evitará que las preguntas resulten incómodas, por lo que se recomienda comenzar con cuestiones que no sean muy directas.

Para evitar la posible inhibición de los hablantes, se han propuesto tareas llamadas Discourse Completion Task (Blum-Kulka et al., 1989), por las que se crea una pequeña situación plausiblemente real y se pide al hablante que responda a ese problema. De la misma forma, se han creado juegos como la tarea del mapa (Anderson et al., 1991), por la que el hablante explica al 
investigador, o a otro hablante, cómo llegar de un sitio a otro. Es una forma de conseguir que los participantes hablen y que todos los discursos sean similares en forma y contenido.

Depende de los objetivos del corpus, en ocasiones el investigador puede estar interesado en ciertos aspectos fonéticos muy concretos, por lo que puede hacer uso simplemente de textos fonéticamente equilibrados que los informantes han de leer (Bruyninckx et al., 1994; Ortega et al., 2000) ${ }^{4}$.

En general, los participantes de los trabajos lingüísticos siempre suelen ser voluntarios que nos prestan su habla pro bono, así que el investigador mostrará siempre agradecimiento, tanto a la hora de recoger los datos, como a la hora de confeccionar el estudio, donde es adecuado dedicar un apartado a estos agradecimientos. En el caso de que los participantes hayan sido remunerados, en algunas ocasiones ocurre, se indicará en el trabajo, de cara a un posible conflicto de intereses, y se agradecerá su participación igualmente.

\section{Recomendaciones sobre las grabaciones}

Como ya se ha puesto de manifiesto en el apartado anterior, sea cual sea el fin de las grabaciones, se tiene que realizar una planificación previa. Por ello, hay que decidir de antemano si es necesaria una grabación de solo audio o de vídeo y audio (más completas que las meras grabaciones de audio), y en función de ello se seleccionará el material adecuado. Todo dependerá del objetivo de la investigación, si no interesa al investigador la recogida de los gestos $O$ no es una investigación de imagen cerebral, para un corpus destinado al análisis fonético bastará con la grabación del audio ${ }^{5}$. No obstante, las grabaciones solo en audio tienen el inconveniente de que si no se entiende algo de lo que se produce, no se cuenta con el referente visual.

En este sentido, si se va a utilizar una grabadora de sonido con el objetivo de realizar un análisis acústico posterior, se necesita que esta sea de la máxima calidad posible: una grabadora especializada que pueda grabar frecuencias altas, ya que las grabadoras pequeñas que usan los periodistas, por ejemplo, no serían suficientes.

En general, si se va a registrar la voz de una sola persona, con un único micrófono, se seleccionará el canal mono y siempre se debe tanto grabar como analizar el sonido en formato wav que es un tipo de archivo que no comprime el sonido. Si se han realizado las grabaciones en mp3, que es un formato de audio comprimido, puede ocurrir que se pierda información en la reconversión, por eso desde el principio hay que seleccionar la opción wav. Normalmente se utiliza una resolución de 16 bits, que se seleccionará previamente en la grabadora. De la misma forma se elegirá una frecuencia de muestreo adecuada. La frecuencia de muestreo es el número de muestras por unidad de tiempo que se toman de una señal de habla real para producir una

\footnotetext{
4 De la misma forma se pueden preparar oraciones determinadas por el objetivo de la investigación y se puede pedir a los informantes que las lean. Normalmente este tipo de práctica se realiza cuando se va a realizar un experimento concreto, no cuando se pretende conseguir un corpus mayor, por las limitaciones obvias.

5 Actualmente se está trabajando con los gestos articulatorios, pero para poder realizar una investigación sobre ellos se necesitará, por ejemplo, hacer uso de articulografía magnética, por lo que la recogida del corpus responderá a unas características específicas.
} 
señal discreta (digital) en el proceso de grabación, ya que conseguir reproducir completamente el habla real sería imposible. Para que se recoja información suficiente la frecuencia con la que se graba ha de ser al menos el doble que la frecuencia de la señal; así que lo más recomendable es que se grabe con una frecuencia de muestreo de 44.100 hercios $^{6}$. Si no se elige una frecuencia suficiente, no se reconstruirá correctamente la señal y en el espectrograma podrán aparecer espacios en blanco ${ }^{7}$.

En cuanto al tipo de micrófono que se va a utilizar, normalmente se prefieren los micrófonos cardioides, que recogen solo la parte de sonido a la que se dirigen, y se suelen colocar a unos $8-10 \mathrm{~cm}$ de la boca del informante. Si se quiere grabar el sonido ambiente entonces se seleccionará un micrófono omnidireccional, que pueda grabar en cualquier dirección y que se colocará en un espacio intermedio entre el investigador y el informante. En los dos casos lo más aconsejable es utilizar un micrófono de mesa o de diadema, ya que un micrófono en la solapa del informante puede introducir el ruido de, por ejemplo, su camisa. Si se elige el de diadema, se intentará que no esté muy cerca de la boca del entrevistador para que no sature la señal, ni las oclusivas $(/ p, t, k /)$ suenen después como choques bruscos en el audio. Asimismo, si el informante sujeta papeles en la mano, tendrá cuidado a la hora de moverlos porque todo esto quedará grabado y si hay ruido, de nuevo, se invalidará el análisis fonético.

También hay que prestar atención a la habitación donde se vaya a grabar: si se trata de una habitación grande, sin cortinas, probablemente habrá bastante eco que quedará reflejado en la grabación. Para evitarlo, se recomienda buscar una habitación pequeña, con cortinas, las cuales puedan absorber las reverberaciones no deseadas y que el micrófono no se sitúe demasiado lejos del entrevistador.

En cualquier caso, micrófonos y grabadoras se prepararán al principio de cualquier tarea para no distraer a los informantes durante su intervención. Y se comprobará siempre en este momento su estado: que estén encendidos, que tengan batería suficiente o pilas de repuesto, que el volumen sea el adecuado, etc. De la misma forma, antes de comenzar la grabación se decidirá dónde se coloca la grabadora y dónde se sitúan los participantes, ya que en función de lo que interese grabar elegiremos el lugar idóneo.

\section{Recomendaciones sobre la confección del corpus}

$Y$, finalmente, con todo el material recogido es el momento de construir el corpus. Como se trata de un corpus oral, donde lo que interesa es mantener las grabaciones originales, lo más importante es que se elija un tipo de soporte que permita alinear el audio con la transcripción del texto que se vaya a realizar. Disponer del audio en un archivo y en otro distinto la transcripción escrita no es demasiado operativo para el análisis fonético, ya que se

\footnotetext{
- Si se va a grabar voz cantada, como la frecuencia de esta señal es mucho más alta que la del habla, la frecuencia de muestreo será superior a $44.100 \mathrm{~Hz}$.

${ }^{7}$ Puede ocurrir que en algunas ocasiones se utilicen programas que no admitan esta calidad de audio y haya que bajar la resolución de los archivos para poder subirlos a ese determinado programa. Es recomendable leer las instrucciones de las herramientas con las que se vaya a trabajar antes de realizar cualquier grabación.
} 
necesitará volver continuamente sobre el archivo sonoro. Por ello, es recomendable elegir cualquiera de las herramientas que ya han sido desarrolladas para este fin. Algunas de ellas se señalan a continuación.

ELAN (Wittenburg et al., 2006) es una herramienta que sirve para alinear texto transcrito con su vídeo correspondiente; creado en el Max Planck Institute for Pyscholinguistics, The Language Archive, Nijmegen (<https://tla.mpi.nl/ tools/tla-tools/elan/>).

Por otro lado, EXMARaLDA (<http://www.exmaralda.org/en>) (Smith et al., $2011)$ permite alinear audio y texto. Se compone de una herramienta de transcripción y anotación y un sistema de análisis y búsqueda. Fue creada dentro del proyecto Computer assisted methods for the creation and analysis of multilingual data en el Centro de Multilingüismo (Sonderforschungsbereich Mehrsprachigkeit - SFB 538) de la Universidad de Hamburgo.

También es destacable Phon (Rose et al., 2006), diseñado específicamente para el trabajo sobre la adquisición fonológica, segmenta archivos de vídeo o audio y estos se pueden alinear con las correspondientes transcripciones. Además, este sistema permite descargar todo el corpus en formato CSV y XML, por lo que los resultados de las búsquedas se pueden exportar a documentos fácilmente. El programa fue creado por Yvan Rose (Memorial University, Canadá) y está disponible en red en el siguiente enlace: (<http://childes.psy.cmu.edu/phon/>).

Cualquiera de ellas, entre otras, permite trabajar con las transcripciones y los archivos originales de manera conjunta, lo que facilitará el análisis fonético. Todas tienen motores de búsqueda basados en algoritmos que pueden reconocer el habla automáticamente, por lo que la transcripción en ocasiones puede ser semiautomática y tienen sistemas de programación que permiten manipular los datos, como por ejemplo, alineamientos automáticos de las fronteras fonéticas en la señal de habla y búsqueda de patrones fonéticos. En general, suelen ser compatibles con Praat (Boersma y Weenink, 2016, version 6.0.17) (<http://www.fon.hum.uva.nl/praat/>) para poder realizar análisis acústicos.

Sea cual sea la herramienta que se elija, son necesarias las transcripciones de la lengua oral. Transcribir consume una gran cantidad de tiempo. MacWhinney (2010) estima que 10 minutos de grabación conllevan 2,5 horas de trabajo para transcribir, lo que supone más de 15 horas de trabajo por cada hora de grabación, incluso llegando hasta 20 horas.

Normalmente se prefiere que el transcriptor sea un hablante nativo que hable la misma lengua del informante y se recomienda que sea el mismo investigador que ha realizado las grabaciones. A la hora de transcribir hay que decidir qué información va a ser relevante, qué es lo que interesa que aparezca. Si el estudio se destina al análisis fonético, como es el caso, resulta fundamental que se realicen transcripciones fonéticas; no es suficiente con que sean ortográficas. Y en el caso de la transcripción fonética se utilizará siempre el alfabeto fonético internacional (API/AFI).

En cuanto a la fiabilidad de los datos, se recomienda que las transcripciones se realicen por un transcriptor y otro investigador diferente actúe de revisor. 
Además, de que para que tenga la suficiente validez científica un $10 \%$ de la transcripción se realizará por otro transcriptor y entre los dos tendrán que alcanzar una tasa de acuerdo del 95\% (Escudero et al., 2012).

Asimismo, una vez transcritos, los textos se pueden anotar o codificar con diferentes etiquetas en función de las necesidades del investigador. En todo caso se recomienda seguir reglas estandarizadas de transcripción y anotación, como las propuestas por TEl (Text Encoding Iniciative, <http://www.teic.org/index.xml>). Y si en algún caso es necesario crear un código propio, este será fácilmente manejable por los transcriptores y los posteriores investigadores que accedan al corpus.

En cualquier caso la tendencia actual, y última recomendación, es publicar los corpus siempre en abierto para que estén a disposición de la comunidad científica. De esta forma estarán disponibles para que cualquiera pueda investigar sobre ellos, lo cual tiene la ventaja de las continuas revisiones que se pueden hacer sobre el propio corpus, tanto de las transcripciones como de las anotaciones y etiquetas; así las mejoras futuras de las herramientas de trabajo mejorarán nuestro conocimiento del lenguaje con unos datos cuya fiabilidad se valida continuamente.

\section{Conclusiones}

En definitiva, en estas páginas se ha tratado de dar una serie de pautas a los futuros investigadores que quieran crear un corpus oral válido para el análisis fonético o acústico. Son consejos que conciernen a los participantes, a las grabaciones y a los sistemas de organización del corpus. Sean cuales sean las decisiones que tome el investigador, tanto su recogida de datos como el tratamiento que de ellos haga después tienen que ser óptimos para que su investigación cumpla los requisitos de calidad requeridos para ser publicada.

\section{Bibliografía}

BLUM-KULKA, S., J. HOUSE \& G. KASPER (1989): Cross-cultural Pragmatics: Requests and Apologies. Norwood, NJ: Alblex Publishing Corporation.

BOERSMA, P. \& D. WEENINK (2016): Praat: Doing Phonetics by Computer, (versión 6.0.17) <http://ddw.praat.org/>.

BRUYNINCKX, M., B. HARMEGNIES, J. LLISTERRI \& D. POCH (1994): LanguageInduced voice quality variability in bilinguals. Journal of Phonetics, 22(1), pp. 19-31.

DURAND, J., U. GUT \& G. KRISTOFFERSEN (eds.) (2014): The Oxford Handbook of Corpus Phonology. Oxford: Oxford University Press.

ESCUDERO, D., L. AGUILAR, M. M. VANRELL \& P. PRIETO (2012): "Analysis of intertranscriber consistency in the Cat_ToBI prosodic labelling system". Speech Communication, 54, pp. 566-582.

ANDERSON, A.H., M. BADER, E.G. BARD, E. BOYLE, G. DOHERTY, S. GARROD, S. ISARD, J. KOWTKO, J. MCALLISTER, J. MILLER, C. SOTILLO, H.S. THOMPSON \& R. WEINERT (1991): "The HCRC Map Task corpus". Language and Speech, 34,4 , pp. 351-366.

KOCK, J. de (2001): Lingüística con corpus. Catorce aplicaciones sobre el español. Salamanca: Universidad de Salamanca. 
KENNEDY, G. D. (1998): An Introduction to Corpus Linguistics. Londres: Longman.

LÓPEZ MORALES, H. (1994): Métodos de investigación lingüística. Salamanca: Colegio de España.

MACWHINNEY, B. (2010): Introduction to CHILDES and TalkBank. Presentación de Powerpoint en la página web: http://childes.psy.cmu.edu/intro/.

MCENERY, T. \& A. WILSON (1996): Corpus Linguistics. Edinburgh: Edinburgh University Press.

ORTEGA, J., J. GONZÁLEZ \& V. MARRERO (2000): "Ahumada: A large corpus in Spanish for speaker characterization and identification". Speech Communication, 31, 2, pp. 255-264.

ROSE, Y., B. MACWHINNEY, R. BYRNE, G. HEDLUND, K. MADDOCKS, P. O'BRIEN \& T. WAREHAM (2006): "Introducing Phon: A Software Solution for the Study of Phonological Acquisition". Proceedings of the 30th Annual Boston University Conference on Language Development. D. BAMMAN, T. MAGNITSKAIA \& C. ZALLER (eds.). Somerville: Cascadilla Press, pp. 489500.

SILVA-CORVALÁN, C. (2001): Sociolingüística y pragmática del español. Washington D.C.: Georgetown University Press.

SCHMIDT, T., K. WÖRNER, H. HEDELAND \& T. LEHMBERG (2011): "New and future developments in EXMARaLDA. Multilingual Resources and Multilingual Applications. Proceedings of GSCL Conference 2011 Hamburg. T. SCHMIDT \& K. WÖRNER (eds.).

SVEC, J.G. \& S. GRANQVIST (2010): "Guidelines for selecting microphones for human voice production research". American Journal of SpeechLanguage Pathology, 19,4, pp. 356-368.

TORRUELLA, J. \& J. LLISTERRI (1999): "Diseño de corpus textuales y orales". Filología e informática. Nuevas tecnologías en los estudios filológicos. J.M. BLECUA, G. CLAVERÍA, C. SÁNCHEZ \& J. TORRUELLA (eds.). Barcelona: Seminario de Filología e Informática, Departamento de Filología Española, Universidad Autónoma de Barcelona - Editorial Milenio, pp. 45-77.

WITTENBURG, P., H. BRUGMAN, A. RUSSEL, A. KLASSMANN \& H. SLOETJES (2006): "ELAN: a Professional Framework for Multimodality Research". Proceedings of LREC 2006, Fifth International Conference on Language Resources and Evaluation. 